\title{
Der Übergang vom Gelehrtenlatein zur deutschen Wissenschaftssprache
}

\author{
Zur frühen deutschen Fachliteratur und Fachsprache in den \\ naturwissenschaftlichen und mathematischen Fächern \\ (ca.1500-1800) [1983/1986]
}

Der Übergang der Wissenschaften vom Lateinischen in die Landessprachen, von der „lingua Europaea universalis et durabilis ad posteritatem“, die Leibniz erhalten wissen wollte, ${ }^{1}$ ins Spanische oder Italienische, Französische, Englische, Niederländische, Deutsche, Schwedische, war ein Vorgang von nicht zu unterschätzender Tragweite und Dynamik. Der Sprachenstreit der frühen Neuzeit zwischen Gelehrtenlatein und Volkssprache ist mit angemessener Heftigkeit geführt worden. Die allmähliche Popularisierung der Wissenschaft in diesem vom Spätmittelalter bis ins 18. Jahrhundert sich erstreckenden Prozess entband Vorzüge und Gefahren der Aufklärung. In dem Augenblick, wo die Wissenschaft landessprachlich wird, wird das öffentliche Weltbild einer wissenschaftlichen Kritik unterzogen, erhält die Wissenschaft $u$. U. einen national bornierten Zug, entsteht eine nationale wissenschaftliche Prosa und Allgemeinbildung und verkommt wissenschaftliche Theorie nicht selten, wie im Fall der Wirkung von Marx, Darwin, Freud, zur politischen Rüstkammer und trivialen Karikatur; wird die öffentliche Gebrauchssprache durch einen breiten Entlehnungsvorgang aus den Wissenschaften bereichert und - belastet. Schon im 18. Jahrhundert beklagte Justus Möser (o.J.: 222f.) in einer seiner Patriotischen Phantasien die Überlastung der Umgangssprache:

[...] es ist gegen die Natur der Sache, unendlich kleinen Teilen und unendlich feinen Unterschieden Größe und Farbe zu geben, daß sie ein jeder sehen und empfinden kann. Außer dem engen Kreis der Wissenschaften verwirret man nur damit den gesunden Menschenverstand. Die ganze Behandlung einer Sache und die zu deren Vortrag gewidmete Sprache wird dadurch entweder zu scharf bestimmt oder zu manigfaltig, um sie zu seinen

1 Gottfried Wilhelm Leibniz: Kurzes wohlgemeintes Bedenken vom Abgang der Studien und wie denenselben zu helfen. Denkschrift 1711. Zit. nach Leibniz 1916a: XXIf.

Anmerkung: Erweiterte Fassung eines Vortrags, der am Berliner Wissenschaftskolleg konzipiert und am 25.5.1983 vor dem Freiburger Linguistenkreis gehalten wurde. Ich danke insbesondere Frau Dr. Eva Schütz für Kritik und Anregung, Herrn Christoph Martin für Hilfe und Hinweise bei der Erstellung des Manuskripts.

Ә Open Access. (C) Uwe Pörksen, publiziert von De Gruyter. (c) BY-NC-ND Dieses Werk ist lizenziert unter der Creative Commons Attribution-NonCommercial-NoDerivatives 4.0 Lizenz. 
ordentlichen Bedürfnissen zu gebrauchen. [...] Das ganze Reich des Unendlichen, was vor unsre Sinnen versteckt liegt, ist überdem das Feld der Spekulation und Systeme. Jeder legt hier sein Eignes an, bestimmt darnach seine Worte oder erfindet für seine Hypothese besondere Zeichen, und wann die gemeine Menschensprache damit überladen wird: so entsteht daraus, eben wie aus einer Menge zu vielerlei Münzen, Beschwerde und Verwirrung; man unterscheidet, wo man nicht unterscheiden sollte, und wird spitzfindig, anstatt brauchbar zu werden; oder ein Mensch versteht den andern nicht mehr; und unsrer jetzigen Sprache wird es wie der ehemaligen scholastischen ergehen, die durch ihre Feinheit verunglückt ist [...].

Wir können davon ausgehen, dass die Erarbeitung der mathematischen, naturkundlichen, medizinischen „Fächer“ in der Landessprache, die im Spätmittelalter beginnt, ein erstrangiger Faktor unserer Sprachentwicklung wurde, seit diese Bereiche im 19. Jahrhundert unsere Geschichte bestimmen. Ihr Übergang in die Landessprache ist aber für die deutschen Verhältnisse bisher nicht oder doch nicht als Ganzes beschrieben worden. Es gibt Einzeluntersuchungen. Unsere Literaturgeschichte berücksichtigt bisher leider kaum die Sachliteratur, und unsere allgemeinen Sprachgeschichten nehmen von den mathematischen, naturwissenschaftlichen, medizinischen Quellen nur am Rande Notiz. - Ist das Material im deutschen Sprachbereich besonders widerspenstig?

Auch das; aber die Gründe für das Versäumnis liegen vermutlich zunächst in der weit zurückreichenden Trennung der von Snow diagnostizierten „Zwei Kulturen“. Die Kluft geht heute vielleicht stärker als jemals durch unsre Gesellschaft: auf der einen Seite existieren die exakten Wissenschaften und ihre breite Anwendung in Technik, Wirtschaft, Volksgesundheit, auf der anderen die Geisteswissenschaften und ihre Pflege meist historischen Überlieferungsgutes. Die Unkenntnis und Furcht vor der jeweils anderen „Kultur“ wachsen auf beiden Seiten. Eine zweite Sprachentrennung, nach der historischen zwischen dem Gelehrtenlatein und der Landessprache, nämlich die rapide Entfernung der naturwissenschaftlichen Fachsprache von der Gemeinsprache bzw. ihr Überschreiten der Grenze zur Nichtsprachlichkeit in den letzten Jahrzehnten haben zur Folge, dass das öffentliche Weltbild heute weit hinter dem der Fachwelt hinterherhinkt und von den Naturwissenschaften des 19. Jahrhunderts bestimmt wird. ${ }^{2}$

Die Kluft zwischen den beiden Welten hat Gründe, die zumindest in die frühe Neuzeit zurückreichen. Die neuzeitliche Naturwissenschaft verselbständigte sich keineswegs nur, indem sie sich von Kirche und Theologie emanzipierte. Sie hatte sich ebenso von der Vorherrschaft des Humanismus zu befreien. Sie stand im

2 Vgl. Ditfurth 1981: „Wir haben die Wissenschaft, die Naturwissenschaft, als Erkenntnisquelle überhaupt noch nicht wirklich zur Kenntnis genommen.“ 
Gegensatz zur humanistischen Wiederbelebung des Lateins, koppelte sich ab von Poesie und Rhetorik, ${ }^{3}$ befreite sich von ihrem aus dem Mittelalter stammenden fast noch philologischen Charakter, verlor ihren theologisch begründeten ästhetischen und kontemplativen Zug. Das geschah überwiegend im 17. Jahrhundert.

Im deutschen Sprachgebiet gab es eine zweite vielleicht noch einschneidendere Auseinanderentwicklung nach 1800. Die empiristische, positivistische, materialistische Naturwissenschaft löste sich von der romantischen Naturphilosophie, entwickelte sich in Auseinandersetzung mit etwas, was ihr, keineswegs immer zu Recht, als rein spekulative Vergewaltigung der Natur erschien. Herder und Goethe, Hegel, Ritter, Schelling und Oken wurden mit zum Anlass einer gründlichen Emanzipation von den Geisteswissenschaften, als sich, zu Beginn des 19. Jahrhunderts, die naturwissenschaftliche Fakultät auch institutionell von der philosophischen Fakultät zu trennen und an den modernen Universitäten ein Eigenleben zu führen begann.

An der Geschichte der Physiologie in Berlin, an Johannes Müller, der als Schüler Goethes begann, nach 1830 zu einem beispielhaften Vertreter der Erfahrungswissenschaften wurde und dessen Schüler Helmholtz und Du Bois-Reymond das mechanistische Weltbild der exakten Disziplinen ausformulierten, ist dieser Vorgang besonders gut zu studieren. ${ }^{4}$

Die Folgen jener Trennung lassen sich zu unserem Nachteil auch in unserem Fach nachweisen. Die Sprachgeschichtsschreibung und Lexikographie der in der Romantik wurzelnden Germanistik wandte sich den alten Sprachdenkmälern und Quellen zu. In den Sprachgeschichten und in der älteren Lexikographie nach Campes Wörterbuch (1807-1811) haben die Sprache der Dichtung, alter Berufsstände und versinkender Techniken und der mundartliche Wortschatz traditionellerweise einen sehr hohen Stellenwert. Die Begriffe der Naturwissenschaften sind schon deshalb oft ausgeschlossen, weil sie nicht dem Erbwortschatz angehören. ${ }^{5}$

Wo ältere Sachprosa untersucht wurde und wird, durch Gerhard Eis und seine Schule (vgl. Eis 1967; 1971; Keil \& Assion 1974; Keil u.a. 1979), wurde eine institutionsgeschichtlich und nicht sachlich begründete Grenze zur Forschungszäsur. Die Altgermanistik ist traditionellerweise zuständig für die Literatur des Mittelalters bis 1500. Eis und seine Schüler, die in der Erschließung der Sachprosa

3 Vgl. Heidelberger \& Thiessen 1981: 223ff., Kap. II, 3 Die Verflechtung mit den rhetorischen Disziplinen, und den 3. Abschnitt dieses Aufsatzes.

4 Mendelsohn 1974: 251, meint, dass die Ablehnung der deutschen Naturphilosophie eine Erklärung für den „Extremismus des Physikalismus und des Materialismus“ vermittelt, der in Deutschland entstand.

5 Vgl. Seibicke in: Drozd \& Seibicke 1973: 4ff.; Seibicke gibt hier zugleich den Hinweis auf das unschätzbare Werk Olschkis 1919/1922/1927. 
Pionierarbeit leisteten und leisten, legten den Schwerpunkt lange aufs Spätmittelalter und ließen die Epoche der frühen Neuzeit weitgehend außer Acht. Die Verhältnisse liegen hier freilich, was die Fülle und Heterogenität des Materials angeht, im deutschen Sprachbereich auch besonders kompliziert. ${ }^{6}$

Für die italienischen Verhältnisse gibt es das vielleicht immer noch nicht genügend bekannte dreibändige Werk von Leonardo Olschki, Geschichte der neusprachlichen wissenschaftlichen Literatur, das zwischen 1919 und 1927 erschien. Das Werk beschreibt die Herausbildung einer volkssprachlichen Literatur auf dem Gebiet der Technik und der Mathematik, der naturwissenschaftlichen Disziplinen und der Medizin - und das alles im Kontext der Ablösung des Lateinischen durch das Italienische. Die italienisch-lateinische Sprachenkonkurrenz ist das Hauptthema des zweiten Bandes, der erste Band stellt Leonardo da Vinci, der dritte Galilei in den Mittelpunkt. Das Werk zu lesen ist ein Vergnügen; es ist auch für uns eine einzigartige Quelle.

Olschki hatte die Absicht, als vierten Teil eine „Geschichte der wissenschaftlichen Literatur in Frankreich“ zu schreiben, die bis ins 18. Jahrhundert reichen sollte, und hatte vergleichbare Pläne für das deutsche Sprachgebiet. Er schreibt im Vorwort des zweiten Bandes:

$\mathrm{Ob}$ es dem Verfasser möglich sein wird, in gleicher Weise eine Literaturgeschichte der Wissenschaften in Deutschland zu entwerfen, kann heute nicht entschieden werden. Der Leser kann sich ein Bild von der großen Schwierigkeit dieser Aufgabe machen, wenn er bedenkt, daß Notker schon um das Jahr 1000 versuchte, eine deutsche Sprache der Wissenschaft zu bilden, während Gauß im 19. Jh. sich im wesentlichen der lateinischen Sprache bediente und die Sitzungsberichte der Berliner Akademie der Wissenschaften jahrzehntelang französisch abgefaßt waren.

Das Vorhaben scheint nicht realisiert worden zu sein. Die dezentralisierte und vielschichtige Geschichte der neusprachlichen wissenschaftlichen Literatur in Deutschland wäre an keine einheitliche und führende Region, an keine Akademiegeschichte anzuschließen, auch nicht an einen einheitlichen Epochenwillen wie in Norditalien seit der Frührenaissance. Und es fehlen so eindrucksvolle Geschichtsordner wie Leonardo und Galilei.

Wollte man eine derartige Geschichte an Personen binden, ließe sich eine Reihe denken, die von Paracelsus über Kepler, Leibniz und Thomasius bis zu

6 Vgl. Kühlmann 1980: Anm. 45: „Zur Auseinandersetzung zwischen Muttersprache und Latein auf diesem Sektor [gemeint ist Technik einschließlich Militärtechnik, Ökonomie und Naturwissenschaft; U.P.] hat bekanntlich L. Olschki wesentliche Grundlinien ausgezogen. [...] Die entsprechenden Prozesse im Deutschland des 17. Jahrhunderts liegen noch weitgehend im Dunkeln, da vor allem das Sachschrifttum außerhalb der Belletristik kaum erschlossen ist.“ 
Christian Wolff führt. Etwas Vergleichbares wäre das nicht; der Eindruck bliebe diffus. Ein andrer Zugang wäre es, von dem seit dem Spätmittelalter nicht abreißenden und sich verbreiternden Strom der praktischen Gebrauchsliteratur auszugehen, der Bergbüchlein, Kräuterbücher, Handreichungen zur Arzneikunde von den Berufssprachen.

Ich will hier in das große Meer, in dem ich schwimme, nur ein paar aphoristisch hingehauchte Inseln setzen.

\section{Die erste Insel: Thomasius und Leibniz}

Gegen die Ostermesse 1687 hing am Schwarzen Brett der Leipziger Universität folgender Anschlag:

Christian Thomas/ eröffnet/ Der Studirenden Jugend/ zu Leipzig/ in einem Discours/ Welcher Gestalt man denen Frantzosen in gemeinem Leben und Wandel nachahmen solle?/ ein COLLEGIUM über des GRATIANS Grund Reguln/ Vernünftig, klug und artig zu leben. - zu finden bei Moritz George Weidemann. ${ }^{7}$

Diese Ankündigung war ein Skandal. Ihr Urheber, der 32-jährige Privatdozent Christian Thomasius, hatte schon vorher Anstoß erregt durch eine Abhandlung über „Vielweiberei“, in der er die Auffassung vertrat, Polygamie verstoße zwar gegen göttliches und menschliches, nicht aber gegen natürliches Recht, und dadurch, dass er nicht im Talar, sondern in Kavalierskleidung in seine Vorlesung ging. Und nun dies.

Denckt doch! [schreibt er nach 30 Jahren als berühmter Gelehrter in Halle] ein teutsch programma an das lateinische schwartze Bret der löbl. Universität. Ein solcher Greuel ist nicht erhöret worden, weil die Universität gestanden. Ich muste damals in Gefahr stehen, dasz man nicht gar solemni processione das löbliche schwartze Bret mit Weyhwasser besprengte. (Zit. nach Hodermann 1891: 18)

Es war in der Tat die erste deutschsprachige Ankündigung am Schwarzen Brett einer deutschen Universität. Vorlesungen in deutscher Sprache hatte es schon vorher vereinzelt gegeben. 150 Jahre früher, 1527, las Paracelsus in Basel über Medizin auf deutsch. Einer seiner Schüler hat die Chirurgia Vulnerum mitgeschrieben, „beides, Lateinisch und Teutsch durcheinander, wie auf etlichen Universite-

7 Das Programm des Thomasius ist mit einem aufschlussreichen Nachwort von Peter von Düffel wieder abgedruckt in einer Reclam-Ausgabe (Thomasius 1970). Zur Einschätzung vgl. auch Grimm 1983: 378f. 
ten in Teutschlandt der Gebrauch desselben Seculi gewesen“, heißt es in der späteren Vorrede. Wie las er?

Wunden ligamentorum possunt curari sine binden (S. 5). Si vis curari, noli sprützen aquam in die Fistell (S. 45). ${ }^{8}$

Paracelsus führt hier den Rezeptstil der Ratgeber des späten Mittelalters fort und durchsetzt sein schweizerisch gefärbtes Deutsch mit lateinischen Wörtern und Fachausdrücken. Er selbst hatte nur sporadisch studiert und war bekanntlich kein Universitätslehrer, sondern ein berühmter und umstrittener Wanderarzt und Schriftsteller. Sein Schrifttum, aus dem Übergangsbereich zwischen Alchemie und Heilkunst, war weitgehend deutsch.

In Basel hatte er kurze Zeit das Amt eines Stadtarztes inne, mit dem die Aufgabe verbunden war, Collegia zu halten. Er zeigte sie am 5. Juni 1527 an durch einen Anschlag. Dieser Anschlag ist freilich lateinisch, eine lateinische „Intimatio“, kein „teutsch Programma“. Darin kündigt er eine Reinigung der Medizin von den historischen Irrtümern an, wendet sich gegen die Autorität des überlieferten schriftlichen Kanons und gegen Rhetorik. Wer ängstlich den Worten Galens, Avicennas und des Hippokrates folge, werde ein hervorragender Doktor, aber kein Arzt. Er setzt Erfahrung und Einblick in Mysterien der Natur und Kenntnis der Heilstoffe gegen schriftliche, rhetorisch versiert wiedergegebene Autorität. Kaum drei Wochen nach der „Intimatio“ verbrannte er auf dem Markt von Basel eine lateinische Medizinenzyklopädie - die Summa von Mesue.

Ich habe die Summa der Bücher in Sankt Johannis Feuer geworfen, auf daß alles Unglück mit dem Rauch in die Luft gang. (Zit. nach Peuckert 1944: 141)

Auch die Fachsprachengeschichte hat ihre Bücherverbrennung.

Paracelsus konnte sich in Basel nicht lange halten, es gab Spottverse, Pasquille von Seiten der Universität - vielleicht wurde die Rivalität zwischen Hochschule und Stadt auf seinem Rücken ausgetragen - er verließ jedenfalls Basel fluchtartig (vgl. Peuckert 1944: 151ff., 161 ff.).

Deutschsprachige Vorlesungen gab es auch nach ihm ganz vereinzelt, aber Thomasius hielt die erste deutschsprachige Vorlesung in einem nicht $\mathrm{zu}$ den Realdisziplinen rechnenden Fach, nämlich in dem hochwürdigen philosophi-

8 Zit. nach Olschki 1919: 446, Anm. 3. Wurde vielleicht doch die Vorlesung lateinisch gehalten und brachte erst die Mitschrift die Sprachenmischung hervor? 
schen Disziplinbereich, ${ }^{9}$ erhob sie, auf der Basis eines neuen Bildungsgedankens, zum Programm und setzte sie durch. Nicht zufällig hatte er seinen ersten deutschen Discours über das Handorakel der Weltklugheit (1647) des spanischen Jesuiten Balthasar Gracián angekündigt. Die Wahl der politischen Klugheitslehre als Textgrundlage markiert eine Neudefinition des kulturtragenden Ideals, die in Frankreich fünfzig Jahre früher vorgegangen war. Das humanistische Milieu hatte dort längst an Prestige verloren gegenüber dem aristokratischen, die Universität und der lateinische Gelehrte waren zurückgetreten vor Hof und Adel. Auch Thomasius wollte an die Stelle des alten Gelehrtenideals das französische Ideal des in der Welt brauchbaren, des weltläufigen „honnête homme“ setzen und, darin Paracelsus vergleichbar, in der Loslösung vom Latein zugleich die Lösung von der tradierten Autorität und ihren Lehrinhalten erreichen (vgl. Grimm 1983: 356ff., 347).

Seine Berufungsinstanz war das „lumen naturale“, das Prinzip einer praktischen Vernunft. In dem Kampf gegen das Lateinische wendet er sich gleichzeitig gegen das alte „aristotelische Orgelwerck“ (Hodermann 1891: 12), gegen eine im überlieferten Lehrkanon erstarrte lateinische Universität: Gelehrsamkeit, Erkenntnis, Bildung seien nicht mehr an die überkommene Gelehrtensprache gebunden, diese sei in dem Zustand, in dem sie zur Zeit gelehrt und gehandhabt werde, sogar eher hinderlich. Thomasius untergräbt die Auffassung von einem Bildungsprivileg der Lateinkundigen. Er wendet sich erklärtermaßen allgemein an den vernünftigen Menschen, gleich welchen Geschlechts und welcher sprachlichen Vorbildung, und will Philosophie, „Weltweisheit“, grundsätzlich allen in ihrer Muttersprache vermitteln. Er erweitert also sein Publikum, wendet sich an eine nicht mehr zunftgebundene breitere Öffentlichkeit - das war auch finanziell interessant -, bezieht auch die „Frauenzimmer“ mit ein und treibt es mit dem Vernunftprinzip so weit, dass er sich bei ihnen für seinen Unterricht sogar besseren Erfolg verspricht, weil sie nicht wie viele Mannspersonen durch den üblichen Lehrkanon und Lateindrill verbildet seien. „Eine neue Schreibetaffel nimmet das jenige so man drauff schreibet gar leicht an [...]. “10 Die Leipziger Universitätskollegen waren aus vielen Gründen zunehmend der Meinung, der Gottseibeiuns habe sich bei ihnen eingenistet. Thomasius entwich nach Brandenburg und erhielt eine Professur in Halle, wo er der in eine Universität umgewandelten Ritterakademie zu großem Ansehen verhalf.

\footnotetext{
9 Vgl. Grimm 1983: 379: „Das Revolutionäre der deutschsprachigen Vorlesungen für die akademische Hierarchie und deren Lehrkanon war die Übernahme von Prinzipien, die den ursprünglich verachteten Realdisziplinen entstammten.“

10 Thomasius 1970: 36; vgl. auch Thomasius 1968 [1692]: Vorrede § 4, § 5; Ausübung der Vernunftlehre, Widmung, $\S \S 37,38,39,125$.
} 
Aus dem gleichen Jahrzehnt wie der Discours, vermutlich aus den Jahren 1682/83, stammt ein Aufsatz von Leibniz, Ermahnung an die Teutsche, ihren verstand und sprache besser zu üben, der ebenfalls den Übergang vom Gelehrtenlatein in die Volkssprache fordert. Leibniz diagnostiziert im Anschluss an die Sprachgesellschaften des Barock eine Sprachkrise - er meint die vielberufene, „erbärmliche“ deutsch-französische „Sprachmengerei“ seiner Epoche -, aber im Gegensatz zu den Sprachgesellschaften sieht er nicht in der Poesie das Mittel, dem Zustand der Sprache aufzuhelfen. Er entdeckt die Bedeutung der Sachprosa. Es bedürfe der Übung, der Ausarbeitung der Sprache in Dingen, die einen „Kern“ in sich haben: in den praktischen Erfahrungsfeldern und Wissenschaften. Er kritisiert die Praxisferne und Betriebsblindheit einer sich in ihre Zunftsprache abschließenden Wissenschaft und fordert die Ausarbeitung von einer Art Kanon der Sachprosa. Er erhebt diese Forderung im Sinn einer allgemein nationalen Aufklärung, des Austausches der geistigen Güter, weil nämlich eine „wohl ausgeübte Muttersprache wie ein rein poliertes Glas gleichsam die Scharfsichtigkeit des Gemüts befördert und dem Verstand eine durchleuchtende Klarheit gibt“ (Leibniz 1983: 63). In einer früheren, lateinisch geschriebenen Denkschrift über die Förderung der angewandten Naturwissenschaften (Consultatio de naturae Cognitione ad vitae usus [...] 1676?) wünschte er die Einführung des Deutschen auch auf diesem Gebiet.

Bei uns gelangen die Leute, auch die lerneifrigen, zu den Naturwissenschaften erst, nachdem sie die Herkulesarbeit der Spracherlernung bewältigt haben, durch die der Geist so abgestumpft wird. [...] Die Wissenschaft ist dem Licht gleich, und es ist ein allgemeines Interesse, daß es auf alle einzelnen ausgegossen sei. (Leibniz 1916a: 86)

In einem späteren Aufsatz, den berühmten Unvorgreiflichen Gedanken (1697), entwickelt Leibniz die Idee einer umfangreichen Wörterbucharbeit; innerhalb dieses Rahmens projektiert er, was bis heute nicht existiert: ein internationales und umfassendes Fachsprachenlexikon, einen Schatz der Kunstwörter. Beides, die „Kernschriften“ und der „Sprachschatz oder cornu copiae“, will er einer neuen Gesellschaft, einer zu gründenden Akademie anvertrauen, in der sich, wie er hofft, die bestehenden Naturwissenschafts- und Sprachgesellschaften vereinigen sollen.

Die deutschen Aufsätze erschienen postum; Leibniz blieb in seinen Veröffentlichungen beim Lateinischen und Französischen. Seine Position in dem Sprachenstreit war zwiespältig. Er war auch im Grundsätzlichen nicht der Ansicht, dass das Lateinische aus Wissenschaft und Unterricht verdrängt werden solle, sondern er wollte, wie eingangs erwähnt, diese ,eine dauerhafte Überlieferung garantierende europäische Universalsprache“ in den Universitäten als Sprache der sogenannten öffentlichen, nicht unbedingt der privaten Vorlesungen erhalten wissen. 


\section{Die zweite Insel: Ein Versuch in Statistik}

Leibniz und Thomasius treten also in den 80er Jahren des 17. Jahrhunderts entschieden für den Übergang vom Lateinischen in eine deutsche Wissenschaftssprache ein, der eine vorwiegend in der Schublade, der andere öffentlich und durch seine eigenen Schriften, z.B. seine zweibändige Vernunftlehre. Wie sah es in der Realität, wie sah es auf dem Buchmarkt aus? Die Forderung, zum Deutschen als Wissenschaftssprache überzugehen, korrespondierte mit der Entwicklung auf dem Büchermarkt. 1681 überragte zum ersten Mal die Zahl der deutschen die der lateinischen Publikationen. Der Übergang insgesamt vollzog sich in einem Zeitraum von etwa 300 Jahren. 1518 war in Deutschland nur $10 \%$ der Buchproduktion deutsch, Ende des 18. Jahrhunderts waren nur noch $4 \%$ oder $5 \%$ lateinisch. So steht es in der Sprachgeschichte von Bach (1965: 309), ohne dass die Quelle für diese Angabe genannt würde.

Eine der möglichen Quellen, aus denen sich eine solche statistische Übersicht gewinnen lässt, sind die Kataloge der halbjährlichen Buchmessen. Rudolf Jentzsch hat schon 1912 die Leipziger Ostermesskataloge der Jahre 1740, 1770 und 1800 untersucht und in ihnen einen Indikator des Epochen- und Publikumswandels entdeckt. Die Erhebungen von Jentzsch (1912: Schlusstabelle) sind für unsere Frage nach der Entwicklung einer deutschen Naturwissenschaftssprache eine bisher ungenutzte Quelle. Es ergibt sich folgendes Bild:

\begin{tabular}{|c|c|c|c|c|c|c|}
\hline \multirow{3}{*}{1740} & \multicolumn{2}{|c|}{$\begin{array}{l}\text { Gesamtzahl } \\
\text { deutsch/lateinisch }\end{array}$} & \multicolumn{2}{|c|}{$\begin{array}{l}\text { Naturwissenschaften } \\
\text { deutsch/lateinisch }\end{array}$} & \multicolumn{2}{|c|}{$\begin{array}{l}\text { Mathematik } \\
\text { deutsch/lateinisch }\end{array}$} \\
\hline & \multicolumn{2}{|c|}{755} & \multicolumn{2}{|c|}{12} & \multicolumn{2}{|c|}{13} \\
\hline & 545 & 209 & 4 & 8 & 10 & 3 \\
\hline \multirow{2}{*}{1770} & \multicolumn{2}{|c|}{1144} & \multicolumn{2}{|c|}{45} & \multicolumn{2}{|c|}{26} \\
\hline & 981 & 163 & 31 & 14 & 23 & 3 \\
\hline \multirow{2}{*}{1800} & \multicolumn{2}{|c|}{2569} & \multicolumn{2}{|c|}{129} & \multicolumn{2}{|c|}{54} \\
\hline & 2442 & 102 & 108 & 21 & 53 & 1 \\
\hline
\end{tabular}

Abb. 1: Der deutsch-lateinische Büchermarkt nach den Leipziger Ostermesskatalogen von 1740, 1770 und 1800 (Jentzsch)

Die Gesamtzahl der Schriften steigt zwischen 1740 und 1800 um das dreieinhalbfache, von 755 Schriften im Jahr 1740 über 1144 im Jahr 1770 bis zu 2569 Schriften im Jahr 1800 - man sieht, eine in bescheidenem Rahmen steigende Flut, die 
allerdings bald $\mathrm{zu}$ der Klage führt, die Nation werde demnächst nur noch aus Schriftstellern bestehen. ${ }^{11}$ Der Büchermarkt, der laut Jentzsch 1740 noch weitgehend für ein gelehrtes Publikum bestimmt ist, erweitert und verbreitert sein Publikum nicht nur auf dem Gebiet der schönen und unterhaltenden, sondern auch auf dem der wissenschaftlichen Literatur, und zwar in der Weise, dass sich „die Grenzen zwischen wissenschaftlich-gelehrten und populären Schriften äußerst schwer, oft überhaupt nicht mehr ziehen“ (Jentzsch 1912: 320) lassen. Der Zug zu den praktischen Lebensbereichen ist stark. Der Erweiterung des Publikums entspricht eine zunehmende Verdeutschung des Büchermarkts. Der Anteil des Lateinischen sinkt von $28 \%$ im Jahr 1740 über $14 \%$ im Jahr 1770 bis auf $4 \%$ im Jahr 1800. Am Rand ist bei Jentzsch auch von den Naturwissenschaften, von Physik, Chemie, Mineralogie, Botanik, Zoologie und von der Mathematik bzw. den Rechenbüchern die Rede. Der Anteil der Naturwissenschaften am gesamten Buchmarkt ist 1740 verschwindend gering (12 von 777 Schriften, 1,5\%) und ihre Sprache noch zu zwei Dritteln lateinisch (8:4). 1770 ist ihr Anteil um das Dreieinhalbfache gestiegen (45 von 1144 Schriften, 4 \%) und ihre Sprache nur noch zu einem Drittel lateinisch (14:31). 1800 hat sich der Anteil der naturwissenschaftlichen Schriften noch einmal verdreifacht (129 von 2569 Schriften, $5 \%$ ), die lateinischen haben sich auf ein Sechstel reduziert (21:108). Die Zeit zwischen 1770 und 1800 ist die Epoche, in der man die lateinisch schreibenden Schweden Linné und Bergman, die Franzosen Bonnet und Buffon ins Deutsche überträgt und wo Adel und Bürgertum, wie wir aus Weimar genau wissen, zu einem naturwissenschaftlichen Publikum werden, wo Goethe Schädelreihen prüft und den Zwischenkieferknochen entdeckt und der Apotheker von Weimar zu chemischen Vorführungen einlädt.

11 Jacobs 1826: 2f. (Vorrede): „Es wird der deutschen Nation oft und nicht mit Unrecht nachgesagt, daß sie sich um ihre besten Schriften, wenn sie einige Jahrzehnte alt geworden, nicht viel mehr bekümmere, als ein leichtsinniger Jüngling um eine alternde Geliebte, und mit so unmäßiger Begierde nach dem Neuen und Neuesten jage, daß die Lebenszeit der Bücher, wie die Dauer der Tage im Christmonat, immer mehr abnimmt, und sich am Ende auf die wenigen Wochen beschränken wird, die zwischen die beyden Culminationspunkte der Literatur, zwischen die Michaelis- und Ostermesse, fallen. Diese Lust an dem Neuen hat, um es beyläufig zu sagen, ihre Licht- und Schatten-Seite. Jene, indem sie die Thätigkeit der Schriftsteller und Modehändler in immerwährender Spannung erhält, was die Bildung der Nation - welche aller Wahrscheinlichkeit nach in kurzem nur aus Schriftstellern und Schriftstellerinnen bestehen wird, auf eine ganz unglaubliche Weise fördern muß, da ja, nach Cicero und Quintillian, Schreiben viel mehr und schneller als Lesen und Hören bildet. Die Schattenseite aber ist, daß es die sehr ehrenwerthe und in vieler Rücksicht ganz vortreffliche Nation, trotz aller Anstrengung, ihres unersättlichen Heishungers wegen, $\mathrm{zu}$ keiner durchgreifenden Bildung, und am allerwenigsten zu einem sicheren Geschmack bringen kann.“ 
Ein wenig anders ist das Bild im mathematischen Fach. Die Zahl der Schriften entspricht um 1740 der Zahl der naturwissenschaftlichen, sie steigt bis 1800 nur proportional zum übrigen Schrifttum, und ihre Sprache ist im Gegensatz zu den naturwissenschaftlichen Schriften schon 1740 fast durchgehend deutsch; in der großen Mehrzahl handelt es sich um praktische Anleitungen und Lehrbücher, z. B. um ein Werk wie Facillima Artis Arithemeticae Methodus. Das ist: Sehr leichter Unterricht und Lehrart der höchst nothwendigsten und nutzbarsten Rechen Kunst von Johann Baptist Lechner, dessen zwanzigste Auflage 1802 erschien. Ein Exemplar dieser Auflage befand sich, um ein nahe gelegenes konkretes Beispiel zu erwähnen, im Besitz des Bauern Johann Jacob Eselgroth aus Eichstetten (Kaiserstuhl). Sein Untertitel: Dieses Werklein bestehet in allen nothwendigen und bestens erklärten Regeln und Exempeln, wie auch in etwas von der kaufmännischen, oder Welsch= und kurzen Praxi, sammt der Quadrat= und Cubik=Wurzel; mit einer Zugabe unterschiedlicher Uhr-Zahlen, und leichtem Unterrichte, wie solche Zahlen auszurechnen sind.

Wir haben einen dreifachen Befund:

- einen relativ bescheidenen Anteil mathematischen und insbesondere naturwissenschaftlichen Schrifttums bis ins 18. Jahrhundert;

- einen frühen Übergang der Mathematik- oder Rechenbücher vom Lateinischen ins Deutsche;

- $\quad$ einen vergleichsweise späten Rückgang des Lateinischen in den Naturwissenschaften.

Die Zahlen sind ein wenig überraschend. Wie erklärt sich der Befund? Lässt er sich erhärten? Man muss zunächst die Frage stellen: Was ist zu zählen? Welche Literatur wollen wir unter dem Begriff ,Naturkunde und Mathematisches` erfassen? Jentzsch projiziert die Fächersystematik von 1910 auf das 18. Jahrhundert zurück und gelangt dadurch zu seinen Resultaten. Das Verfahren ist aber problematisch; die Fächersystematik der alten Universität und das Bewusstsein von einer Fächersystematik war vom Spätmittelalter bis ins 18. und 19. Jahrhundert ein ganz anderes. Gerade dieses Gebiet scheint mir sehr kompliziert und wenig geklärt zu sein; eine übersichtliche Darstellung fehlt anscheinend.

Man muss sich klarmachen: wir haben bis ins 18. Jahrhundert die Vierfakultäten-Universität, bestehend aus Theologie, Philosophie, Jura und Medizin. Erst im 19. Jahrhundert kamen die Naturwissenschaften allmählich als fünfte Fakultät hinzu; in Freiburg spalteten sie sich erst 1910 von der Philosophischen Fakultät ab (vgl. Jäger 1982: 132). Sie waren vorher meist nicht einmal selbständige Lehrfächer.

In den aus den Domschulen des Mittelalters hervorgegangenen Universitäten ist die Naturkunde den anderen Fakultäten ein- und untergeordnet. Ein Student 
lernt sie, vom Mittelalter bis ins 18. Jahrhundert, selbstverständlich in lateinischer Sprache kennen als eine Reihe kanonisierter Texte. Er begegnet ihr schon im Grundstudium, in den „septem artes liberales“ oder der sog. „Artistenfakultät“, und zwar schon in den formalen Fächern des „Triviums“ - die klassischen Poeten und Rhetoriklehrer waren Autoritäten auf dem Gebiet der Naturkunde und dann in einem mehr oder weniger fest umrissenen Autoritätenkanon der Fächer des „Quadriviums“: in der Arithmetik, Geometrie, Astronomie und Musik. Ein Oxforder Student des 13. Jahrhunderts studierte z.B. im Rahmen der „artes liberales“ laut Lehrplan die Physica, Meteorologica, De caelo, De motu animalium von Aristoteles und andere Werke der bis ins 18. Jahrhundert sog. ,Naturphilosophie‘ (vgl. Heidelberger \& Thiessen 1981: 185). Er begegnete der Naturkunde, wenn er am Ende des Grundstudiums als Baccalaureus ein Philosophiestudium wählte, in einigen Bereichen der Philosophie. Er berührte sie in der Theologie. Theologie als ,Königin der Wissenschaften“ definierte den Auslegungshorizont der Natur. Vor allem begegnete er der überlieferten Naturkunde im Rahmen der Medizin; seit Ende des 17. Jahrhunderts beginnen sich die beschreibenden Naturwissenschaften, Chemie, Mineralogie, Botanik, Zoologie, von der Medizin zu emanzipieren. Auch Physik wird gelegentlich selbstständiges Lehrfach. Alles das ist i.e. nirgends übersichtlich dargestellt. Linné, der seit 1735, seit Erscheinen seines Systema Naturae, die beschreibenden Naturwissenschaften des 18. Jahrhunderts beherrschte und am meisten zur Verselbständigung der Disziplinen Mineralogie, Botanik, Zoologie beitrug, lehrte als Doktor der Medizin und schrieb lateinisch.

Ein Autoritätenkanon in lateinischer Sprache, das war jahrhundertelang im Universitätsbereich die Naturkunde. Der Begriff vom Gelehrtenlatein verband sich auch auf diesem Gebiet mit der Vorstellung von autoritätsbesetzter schriftlicher Überlieferung, von Dauer und Trägheit der einmal eingeführten Sprache, und von Universalität.

Auch die wissenschaftliche Mathematik und Astronomie, im Quadrivium verankert, ist lange lateinisch geblieben. Kopernikus (1473-1543) im 16. Jahrhundert, Johannes Kepler (1571-1630) im 17., Leonard Euler (1707-1783) im 18. und selbst noch Karl Friedrich Gauß (1777-1855) im 19. Jahrhundert verfassten ihre Hauptwerke lateinisch. Gegenüber der kleinen Zahl wissenschaftlicher Publikationen machen sich aber früh die praktischen Bedürfnisse geltend. Es gibt seit dem späten Mittelalter Anleitungen zur Rechenkunst und Messkunst in deutscher Sprache. Wie im Bereich der Naturkunde dürfen wir hier mit einem sich verbreiternden Strom von „Kunstbüchern“ rechnen. Wir kommen darauf zurück.

Wie steht es mit der Repräsentanz der Messkataloge, spiegeln sie tatsächlich den Buchmarkt? Geht aus ihnen die jährliche Produktion hervor, der Charakter der einzelnen Bücher, ihre systematische Zugehörigkeit? Schon Jentzsch ist sich 
der Auswertungsprobleme durchaus bewusst, ${ }^{12}$ er hat das Untersuchungsmaterial, die drei Messkataloge, überaus sorgfältig analysiert und gruppiert und hält an dem hohen „sozialpsychischen“ (Jentzsch 1912: 2, 10) Indikationswert der Messkataloge fest. „Denn wenn wir den MK - in früheren Jahrhunderten den Frankfurter, dann den Frankfurter und Leipziger nebeneinander und schließlich den Leipziger allein - als das hinnehmen, was er ist, als ein Verzeichnis der Bücher, die man für wert erachtete, nach den großen Buchhändlermessen zu bringen, und die man von da weiter zu verbreiten wünschte; wenn wir ferner wissen, daß nur eine bestimmte, höhere literarische Aufnahmeschicht als Publikum in Betracht kommt: so bleibt der MK als solcher schon wertvoll genug“ (Jentzsch 1912: 8). Sein Hinweis auf den Aussagewert der Kataloge für das „Gesamtbewußtsein einer Zeit“ (Jentzsch 1912: 13f.) deutet voraus auf unsere heutige Mentalitätsgeschichte.

Wie weit lassen sich die älteren Messkataloge des 16. und 17. Jahrhunderts zumindest als Indikatoren für den Übergang der naturkundlichen und mathematischen Fächer vom Lateinischen ins Deutsche auswerten? Aus der Arbeit am Verzeichnis der im deutschen Sprachbereich erschienenen Drucke des 16. Jahrhunderts, die 1969 in München und Wolfenbüttel aufgenommen wurde, lässt sich einiges über die Unsicherheit unseres Kenntnisstandes entnehmen: die Schätzungen der Zahl von Drucken im deutschsprachigen Raum schwanken für das 16. Jahrhundert noch 1974 zwischen 80.000 und 200.000, und auf einen systematischen Katalog wurde angesichts der fernen Wissenschaftskategorien des 16. Jahrhunderts von vornherein verzichtet (vgl. Bezzel 1974: 179, 183).

Peter Düsterdieck (1974: 218), der die Messkataloge der Jahre 1637 und 1658 untersucht hat, bemerkt abschließend: „Die Meßkataloge des 17. Jahrhunderts sind kein Spiegel der zeitgenössischen deutschen Buchproduktion.“ „Sämtliche Zahlen über die Höhe der deutschen Buchproduktion, soweit sie durch die Meßkataloge greifbar ist, bedürfen der Überprüfung.“ „Der Anteil der Buchproduktion, der auf den Buchmessen angeboten wurde, ist erheblich kleiner, als bis jetzt vermutet wurde. Von der Literatur, die auf den Messen gehandelt wurde, ist nur etwa die Hälfte heute noch als vorhanden nachweisbar.“ In Düsterdiecks Analyse

12 Jentzsch 1912: 7f.: „Aber alles, was dabei geltend gemacht wird: daß die in den MK angegebenen Verleger oft gar nicht die tatsächlichen Verleger, sondern nur die Kommissionsverleger seien, ferner, daß die süddeutsche Literatur nur zu einem geringen Teil in den MK aufgenommen sei, daß die verzeichneten Bücher oft gar nicht neue Produktion oder neue Auflagen seien: alles dies richtet sich nur gegen eine Verwertung, die mit Hilfe des MK zu sicheren Ergebnissen über buchhandelsgeschichtliche Fragen oder zu solchen über die gesamte literarische Produktion Deutschlands in absolut-quantitativer Hinsicht gelangen will; dagegen wird durch diese Einwände nicht angefochten die Verwertung zur Untersuchung der qualitativen Zusammensetzung des im MK vereinigten Büchermarkts [...].“Vgl. die Bemerkungen zur Fächersystematik, ebd.: 11f. 
kommen die uns hier interessierenden Fächer zwar nicht vor, aber für das allgemeine Verhältnis des Lateinischen zum Deutschen lässt sich doch ein Ergebnis entnehmen. Von den 1637 erfassten 575 Büchern sind 304 in lateinischer Sprache erschienen (davon 163 erhalten), 260 in deutscher Sprache (davon 114 erhalten), 11 in andern Sprachen (davon 4 erhalten). Von den 1658 erfassten 702 Büchern sind 402 in lateinischer Sprache erschienen (davon 279 erhalten), 275 in deutscher Sprache (davon 146 erhalten), 17 in andern Sprachen (davon 9 erhalten). Das Lateinische hat in der Mitte des 17. Jahrhunderts noch deutlich das Übergewicht.

Unternehmen wir einen neuen statistischen Versuch, der von der Fächereinteilung der Epoche ausgeht: Suchen wir, unter dem Blickwinkel unserer Fragestellung, einen Überblick über die uns interessierenden Bestände in einer alten repräsentativen Bibliothek zu gewinnen. Die Herzog August Bibliothek in Wolfenbüttel verfügt bekanntlich über sehr umfangreiche Bestände mathematisch-naturwissenschaftlich-medizinischer Literatur aus dem 16. bis 18. Jahrhundert. Lässt sich der alte, historische Bestand nach deutschem und lateinischem Schrifttum mit einigermaßen begrenztem Arbeitsaufwand trennen? Gewinnen wir aus den vorhandenen Verzeichnissen ein klares Bild von der Fächersystematik des 16., 17., 18. Jahrhunderts ${ }^{13}$

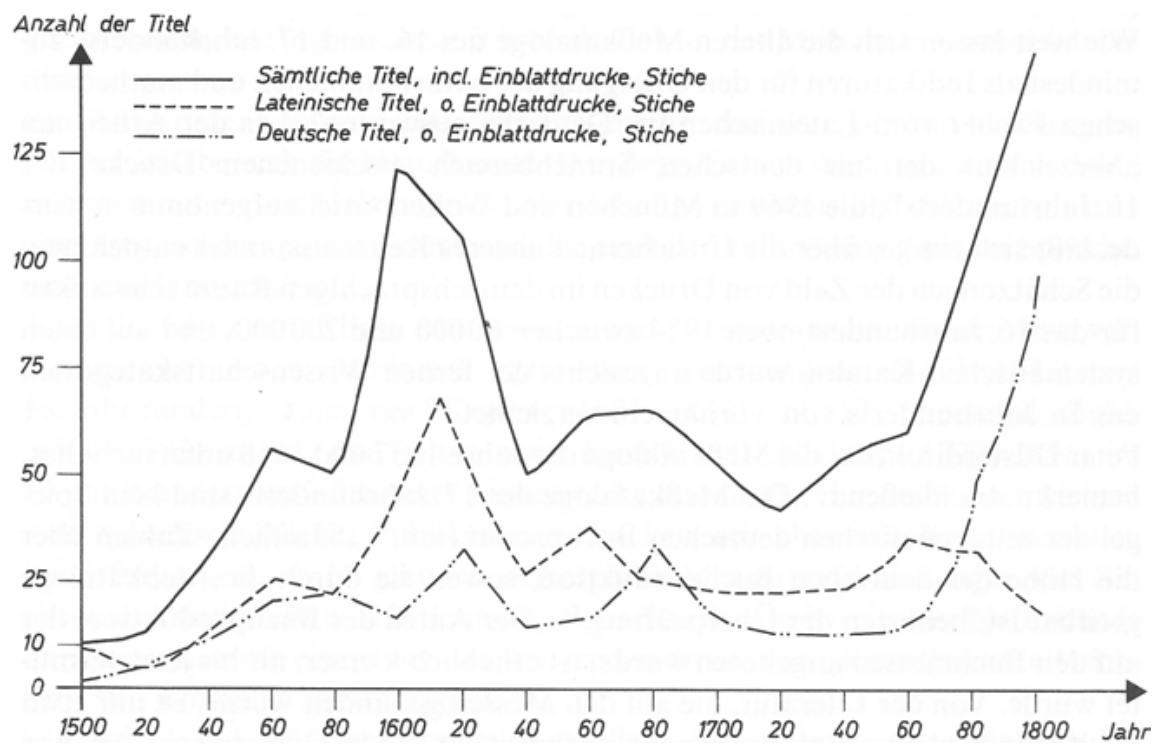

Abb. 2: Lateinische und deutsche Titel 1500-1800 in der Herzog August Bibliothek Wolfenbüttel

13 Mein Mitarbeiter Christoph Martin (M. A.) ist dieser Frage an Ort und Stelle, in Wolfenbüttel, nachgegangen und hat sie konkretisiert. Die beigefügte Graphik stammt von ihm. 
Die hier vorgelegte Graphik beruht auf dem Wolfenbütteler Verzeichnis medizinischer und naturwissenschaftlicher Drucke. 1472-1831, das seit 1976 erscheint, alphabetisch, chronologisch, nach Erscheinungsorten und systematisch, in 4 Reihen also, geordnet. Der Systematische Index (Reihe D, Bd.11-14) ist bedauerlicherweise noch nicht erschienen. Aber auch der 1976 erschienene Chronologische Index (Reihe B, Bd.5-7) ist aussagekräftig. Er verzeichnet die Wolfenbütteler Bestände nach ihrem Erscheinungsjahr. Könnte sich nicht an ihm ein Index für die Relation des Deutschen und Lateinischen gewinnen lassen, in dem man die deutschen und lateinischen Anschaffungen in Abständen von 20 Jahren auszählt? Das Verfahren ist einfach, die Durchschnittszahl der Anschaffungen (wie der Bücherproduktion überhaupt) ist in der Zeit ja durchaus überschaubar. Das Material ist historisch adäquater zusammengefasst, weiter, offener, weniger systematisch.

Der Index verzeichnet Naturwissenschaftlich-Medizinisches im weiteren Sinne: die älteren Signaturen des aufgenommenen Bestandes führen als Stichworte: „Astr., Geom., Arithm., Phys., Bell. (Kriegskunde), Quod. (Verschiedenes), Oec., Math.“ - Bereiche wie Philosophie, Religion, Magie, „artes mechanicae“ schienen der Bearbeiterin Ursula Zachert wohl zu Recht in vielen Fällen nicht recht ausgrenzbar. Man findet in diesem Verzeichnis auch Titel wie Traumbuch Aposomaris (1665), Erinnerung aus Gottes Wort bey dem neuen Comet Stern oder Kurtze Erklärung über die höllische Göttin Proserpina. Das nach Titeln sortierte Material mag gelegentlich ein Werk enthalten, das unter verschiedenem Werktitel mehrfach auftritt. Die Sprache des Werks ist in einigen wenigen Fällen nicht sicher auszumachen. Stiche und Einblattdrucke, die in dieser Zählung ausgeklammert werden sollten, waren nicht in jedem Fall deutlich als solche zu erkennen. Daraus resultieren für die Statistik gewisse Grauzonen, die aber schmal bleiben, da die Zahl der genannten Fälle anscheinend gering ist. Im Übrigen ergibt sich folgendes, recht eindrucksvolles Bild:

Die Zahl der deutschen und lateinischen Bestände im Bereich von Naturkunde und Medizin ist schon im Jahr 1520 gleich groß, läuft bis 1580 etwa parallel, danach überwiegt fast hundert Jahre lang das Lateinische deutlich das Deutsche. Einem allgemeinen Anschaffungshoch um 1620 folgt, aus leicht einsehbaren Gründen, ein Tief um 1640. Leibniz und Thomasius trafen mit ihrer Forderung, ins Deutsche überzugehen, um 1680 den Trend auf dem Buchmarkt. Was vielleicht überrascht, ist, dass bis in die Jahre zwischen 1700 und 1770 nochmals ein deutliches Übergewicht des Lateinischen fällt. Wir müssen berücksichtigen, dass die Medizin in der Sprachenfrage relativ konservativ war; die besondere Geschichte der Herzog August Bibliothek in Wolfenbüttel und ihre Anschaffungspraxis dürfte sich in der Graphik spiegeln. Dennoch mag die langwährende Parallelität der konkurrierenden Sprachen, der späte Niedergang des Lateini- 
schen gegen Ende des 18. Jahrhunderts und die rapide Aufwärtsentwicklung des Deutschen um die gleiche Zeit ein zutreffendes Bild von der Entwicklung im naturwissenschaftlichen und medizinischen Bereich bieten.

Wie sind die Verhältnisse in den europäischen Ländern, wenn man nach der Sprache ihrer führenden Wissenschaftler fragt? Welche Bedeutung haben die Akademien für den Übergang der Wissenschaften in die Landessprache?

\section{Die dritte Insel: Das europäische Umfeld}

Schaut man sich um nach den Verhältnissen in anderen europäischen Ländern, so erscheinen die Akademien als maßgebliche Sachwalter des Übergangs in die Landessprache. Das ist besonders auffällig in Italien. In Florenz entstand aus einem lockeren Kreis literarisch interessierter Bürger, die etwas für die Laisierung der Bildung tun wollten, eine Akademie. „Der Charakter dieser Akademie war ein antiakademischer“, schreibt Olschki, ,ihre ursprüngliche Aufgabe die Vulgarisierung der Bildung." Sie unterstellte sich der Schirmherrschaft Cosimo I., der sich die in der Bürgerschaft von Florenz vorhandenen Tendenzen, die Bildung zu demokratisieren, zunutze machte, um als Herrscher anerkannt zu werden. Cosimo trieb eine volkstümliche Kulturpolitik. Es war der vornehmste Gedanke des Fürsten, berichtet der Historiker der Akademie, das Toskanische zur Gelehrtensprache zu machen:

Er befahl, dass die öffentlichen Vorträge nicht mehr in seiner Residenz, sondern in der Aula der Universität stattfinden sollten und verlangte von den Professoren, dass sie ihre ,publice‘ in der Vulgärsprache abhalten und ihren Volksvorlesungen eine Stelle der Göttlichen Komödie, je nach dem von ihnen vertretenen Fache, zugrunde legen sollten. Diese Vorträge müssten in einem regelmäßigen Turnus stattfinden. Gleichzeitig sollte die Akademie dafür Sorge tragen, dass alle in griechischer und lateinischer Sprache abgefassten wissenschaftlichen und gelehrten Werke ins Italienische übersetzt würden. Nachdem die öffentlichen Vorlesungen mit einem unglaublichen Volkszulauf begonnen hatten, wurde die Akademie am 25. März 1541 feierlich eingeweiht.

Die „Accademia della Crusca“ spaltete sich später von ihr ab - als eine Art Philologenverband (vgl. Olschki 1922: 172, 174).

Die Gründung der Akademie ist also in Florenz fast identisch mit dem Übergang der Wissenschaft in die Landessprache, mit ihrer Popularisierung und Nationalisierung, mit Demokratisierung. Und diese wird bewerkstelligt im Interesse des modernen Staates, der seine Herrschaft $\mathrm{zu}$ festigen strebt und deshalb den in Klostermauern abgeschotteten Geist in Allgemeinbildung umzumünzen vorschlägt. Das Thema des Gegensatzes zwischen modernem Staat und alter 
humanistischer Gelehrtenwelt wird schon bei der Gründung der Florentinischen Akademie angeschlagen; es setzt sich fort, wenn der Staat in Frankreich und England insbesondere die profanen, naturwissenschaftlichen und technischen Fächer stützt, nicht zuletzt aus Gründen ihrer praktischen Nutzung. Auch hier knüpft sich an die Akademiegründung jeweils ein Popularisierungsschub, der dazu gedient haben mag, die neuen Disziplinen in der Öffentlichkeit zu verankern, sie durch das Echo in der Gesellschaft zu legitimieren und abzustützen. ${ }^{14}$

Die 1666 von Ludwig XIV. und Colbert gegründete „Académie des Sciences“ stellte sich auf Wunsch des Königs die Aufgabe, sich auf die zentralen Probleme der Zeit zu konzentrieren, das hieß: auf Pumptechnik und Hydraulik, Schießtechnik und Schifffahrt, Verbesserung von Handwerksgeräten und Maschinen. Der praktischen Zielsetzung entsprach, dass diese Akademie die erste nicht lateinische Gelehrtenzeitschrift herausgab, das Journal des Sçavans (seit 1665). Ihr langjähriger Sekretär Bernhard le Bovier de Fontenelle veröffentliche 1686 seine Unterhaltungen über die Vielzahl der Welten (frz. Entretiens sur la pluralité des mondes), in denen der Gelehrte eine schöne Dame angesichts des Sternenhimmels in die nachkopernikanische Astronomie einweiht, ein Buch, dessen gelehrter Inhalt, dem Journal des Sçavans zufolge, bis in den letzten Winkel und in die Kabinette der Damen vordrang und das in Deutschland, in der Übersetzung von Gottsched (1726), eine Reihe von Auflagen erlebte (vgl. Heidelberger \& Thiessen 1981: 241).

Nationales Prestige, ökonomische und technologische Interessen standen auch Pate bei der Gründung der „Royal Society of London for the Improvement of Natural Knowledge“ von 1662. Der Staat blieb hier mehr im Hintergrund, hatte nur eine allgemeine Schirmherrschaft, aber die Akademie wurde doch durchaus in nationaler Rivalität zu Frankreich gesehen, ihr erster Historiker Thomas Sprat hält es seinem König zugute, dass er die Royal Society noch vor der Académie des Sciences gegründet hatte und sah die „Glorie“ Englands auch in ihren Errungenschaften begründet. Das Organ der Royal Society, die Philosophical Transactions, trat allerdings bedauerlicherweise erst zwei Monate nach dem Journal des Sçavans ans Licht. Thomas Sprat (1616-1713) geht in seiner History of the Royal Society of London for the Improvement of Natural Knowledge, die zuerst 1667, also

14 Mendelsohn 1974: 244, beschreibt den Zusammenhang zwischen der Professionalisierung der Naturwissenschaften und ihrer Popularisierung im 19. Jahrhundert: „Diese Vorlesungen schienen zumindest zwei Funktionen über die offenkundige der gemeinsamen Teilhabe an Information hinaus zu dienen. Erstens hatten sie die Wirkung, das Reservoir zu vergrößern, von dem neue Kräfte für Wissenschaft rekrutiert werden konnte; zweitens erweiterten sie das Publikum, von dem Legitimation und Unterstützung zu suchen war.“ 
fünf Jahre nach der Gründung, erschien, auch auf das Stilideal ein. Man entnimmt ihm den antihumanistischen Affekt, das Antiakademische, Praktische, die Neigung zur Abkoppelung von Poesie und Rhetorik, die auch in Frankreich als naturwissenschaftliches Stilideal galt: Die Royal Society beschloss,

alle Umschreibungen, Abschweifungen und Schwülstigkeiten des Stils zu verbannen und zu jener ursprünglichen Reinheit und Kürze zurückzukehren, mit der die Menschen so viele Dinge mit einer fast gleichen Anzahl von Worten ausdrücken konnten. Sie verpflichtete ihre Mitglieder auf einen präzisen, nüchternen, ungezwungenen Stil, auf konkrete Ausdrücke, klare Bedeutungen und eine natürliche Leichtigkeit, die alles so weit wie möglich der mathematischen Klarheit annähert und sich lieber der Sprache der Handwerker, Bauern und Kaufleute bedient als der der geistreichen Herren und Gelehrten. (Zit. nach Heidelberger \& Thiessen 1981: 243)

Der Reihenfolge der Länder, die soeben eingehalten wurde, entspricht vielleicht überhaupt der des Übergangs ihrer namhaften Mathematiker und Naturwissenschaftler in die Landessprache. Wenn es gestattet ist, ein so triviales Bild zu gebrauchen: Italien liegt deutlich vorne, gefolgt von Frankreich. England behauptet das Mittelfeld. Deutschland schließt auf zu Beginn des 18. Jahrhunderts. In Schweden wird auch im 18. Jahrhundert noch lateinisch geschrieben, in Polen gelegentlich noch im 20. Jahrhundert.

In der europäischen Geschichte des Übergangs vom Lateinischen in die Landessprache war es vermutlich das bedeutsamste Faktum, dass Galileo Galilei, nachdem er seine ersten Werke lateinisch publiziert hatte, im zweiten Jahrzehnt des 17. Jahrhunderts, als das kopernikanische System ihm klar vor Augen stand, zum Italienischen überging.

„Für Bruno und Galilei bedeutet der Bruch mit dem Latein auch den Bruch mit den herrschenden Denkgewohnheiten, die deutlichste Scheidung der alten von der neuen Welt“, meint Olschki. Es war der aufklärerisch-publizistische Eifer, der aus ihnen Märtyrer machte. „Die gesamte [...] philosophische und naturwissenschaftliche Originalliteratur der Renaissance, bis auf Giordano Bruno und Galilei, ist lateinisch abgefaßt.“

In der zweiten Hälfte des 16. Jahrhunderts, wo es wieder vordrang, hat das Lateinische sich sogar in der Geschichtsschreibung wieder durchgesetzt.

Um so höher ist die kühne Tat Brunos und Galileis zu bewerten, mit dieser Sitte zu brechen. Denn das Latein war nicht nur, wie im Mittelalter, die Sprache der Bildung, die offizielle Sprache der Kultur, sondern auch der schützende Wall der kühneren Denker. [...] Bruno und Galilei wurden, wie Calvin, erst dann verfolgt und verurteilt, als sie ihre Ideen und Entdeckungen in gemeinverständlicher Form zu verkünden wagten. Man betrachtete demnach die geistigen Kämpfe und Auseinandersetzungen als eine innere Angelegenheit der Gelehr- 
tenrepublik. [...] In lateinischer Sprache konnten die kühnsten Gedanken dem Schutze der Geistlichkeit anempfohlen werden. ${ }^{15}$

Erst durch den Übergang in die Landessprache wird das allgemein anerkannte Weltbild einer wissenschaftlichen Kritik unterworfen und die gelehrte Publikation zum Skandal. Die wissenschaftliche Wahrheit verändert ihren Charakter. Das lässt sich wiederholt, z.B. auch an Paracelsus und Thomasius, beobachten. - Der Fall Galilei enthüllt auch die Verlustseiten jenes Übergangs. Die Wahl der Landessprache entfremdet ihn ebenbürtigen Gesprächspartnern in anderen Ländern; mit ihr verband er die Form des Dialogs, der an gesprochene zeitgenössische Dispute anknüpfte. Diese Form irritierte Descartes und veranlasste ihn zu ausfälligen Bemerkungen gegen Galilei; ,die literarische Aufmachung mathematischer Werke [...] erschien ihm ein Verbrechen gegen den heiligen Geist der Mathematik“ (vgl. Olschki 1922: 109; Olschki 1927: 292, 338-348, 353). Und Johannes Kepler beklagte sich über Galileis Wahl der Muttersprache. Er verstand nicht Italienisch. Die europäische Universalsprache und die in ihr realisierte geistige Gemeinschaft der Gelehrten zerbrach. Kepler sprach von einem „crimen laesae humanitatis““ ${ }^{16}$

Mit Galilei ging selbstverständlich nicht die italienische Wissenschaft in die Landessprache über; der Anatom Galvani z.B. veröffentlichte seine Abhandlung über tierische Elektrizität 1791 lateinisch. Wir haben mit einem auch innerhalb der einzelnen europäischen Länder ungleichzeitigen Vorgang zu rechnen und müssen nach Disziplinen, nach Schreibanlass und Zielpublikum des jeweiligen Werks unterscheiden.

Versuchen wir dennoch, da einschlägige Sprachgeschichten z.B. Frankreichs oder Englands keine Auskunft geben, uns von dem Vorgang in einigen europäischen Ländern ein Bild zu machen, indem wir von einer Reihe bekannter Mathematiker und Naturforscher des 16. bis 18. Jahrhunderts ausgehen. Unsere Liste ist ganz unvollständig, das Folgende ist, wie diese Arbeit im Ganzen, ein fragmentarischer Entwurf.

In Frankreich gilt Norditalien früh als Vorbild. Das zeigt die Geschichte der „Pléiade“ im 16. und die der „Französischen Akademie“ im 17. Jahrhundert. Die bekannteren Autoren schreiben im 17. Jahrhundert überwiegend französisch,

15 Olschki 1922: 63f., 66f. - Olschki weist zum Beispiel darauf hin, aus den Prozessakten gehe hervor, dass der Gebrauch der Vulgärsprache bei der Darlegung ketzerischer Lehren als erschwerender Umstand galt. - Galileis Sidereus Nuncius, der seine bedeutendsten astronomischen Entdeckungen veröffentlichte, erschien 1610 mit der Zustimmung zweier Inquisitoren. Vgl. Olschki 1922: 67.

16 Vgl. Olschki 1922: 71. Zum Begriff der ,humanitas‘ als dem ,Commune humanitas corpus‘

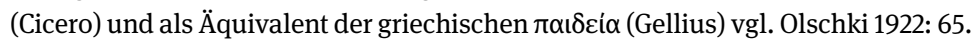


René Descartes (1596-1650) und Blaise Pascal (1623-1662) noch in beiden Sprachen, lateinisch und französisch, aber Edme Mariotte (1620-1684), Pierre Bayle (1647-1706), Bernard le Bovier de Fontenelle (1657-1757), Pierre Luis Moreau de Maupertuis (1698-1759), Bernard de Jussieu (1699-1777), Jean le Rond d'Alembert (1717-1783), Antoine Laurent Lavoisier (1743-1794), Jean Baptiste Lamarck (1744-1829), Pierre Simon de Laplace (1749-1827), Georges Cuvier (1769-1832), also Physiker, Enzyklopädisten, Mathematiker, Botaniker, Chemiker, Zoologen, veröffentlichen französisch. Maupertuis, der Mitte des 18. Jahrhunderts in Berlin lebte und acht Jahre lang Präsident der Berliner Akademie war, machte dort freilich den Vorschlag, eine Stadt zu gründen, in der nur Lateinisch gesprochen werden sollte, und provozierte damit den Spott Voltaires, die Diatribe du Docteur Akakia, die zu dem sog. „Potsdamer Skandal“ führte (vgl. Olschki 1922: 72).

In England bleibt das Lateinische länger erhalten, der Übergang in die Landessprache erfolgt anscheinend während des 17. Jahrhunderts. William Gilbert (1540-1603) schreibt seine Abhandlung über den Magneten lateinisch, Francis Bacon (1561-1626) verfasst sein Novum Organum und anderes lateinisch, aber zum Beispiel ein Advancement of Learning (1605) zunächst auf englisch und dann, als De Augmentis Scientiarum (1623), auf lateinisch. William Harvey (15781657) schrieb seine Arbeiten über den Blutkreislauf lateinisch (1628), eine erhaltene Mitschrift seiner Anatomievorlesung von 1616 zeigt die uns von Paracelsus bekannte Sprachenmischung: „W.H. (William Harvey) constat per fabricam cordis sanguinem per pulmones in aorta perpetua transferri, as by 2 clacks of water bellow to rayse water“ (zit. nach Mason 1961: 268; vgl. Anm. 8).

Robert Boyle (1627-1691) schrieb überwiegend englisch, Robert Hooke (16351703) lateinisch und englisch, Isaac Newton (1642-1727) veröffentlichte seine Mechanik, die Philosophiae naturalis principia mathematica, 1687 auf lateinisch, seine Optik, die Opticks or, a treatise of the reflexions, refractions, inflexions and colours of light, 1704 auf englisch. Stephan Hales (1677-1761), Erasmus Darwin (1731-1802), Joseph Priestley (1733-1804), Pflanzenphysiologen, Botaniker, Physiker, schrieben englisch.

Wir übergehen die Niederlande, die als Ort der Naturforschung wie des Drucks anderwärts verbotener Werke seit dem 17. Jahrhundert von großer Bedeutung sind und in denen seit dem 17. Jahrhundert auf lateinisch (Snellius, 15911621), französisch und lateinisch (Christian Huygens, 1629-1695) oder lateinisch und holländisch (Jan Swammerdam, 1637-1685) veröffentlicht wird, und gehen noch kurz auf die Entwicklung in Deutschland und Schweden ein.

Im deutschen Sprachbereich herrscht im 17. Jahrhundert noch das Lateinische fast ausnahmslos vor, im 18. hat es bis in die zweite Hälfte des Jahrhunderts noch ein starkes Gewicht. Johannes Kepler (1571-1630), Otto von Guericke (16021686), Kaspar Schott (1608-1666), Georg Ernst Stahl (1660-1734), Camerarius 
(1665-1721), Mathematiker, Physiker, Chemiker, Botaniker des 17. Jahrhunderts also, veröffentlichten lateinisch. Ebenso die Mathematiker des 18. Jahrhunderts, die teils in St. Petersburg oder in Basel lehrten, Daniel Bernoulli (1700-1782), Leonard Euler (1707-1783), Johann Bernoulli (1710-1790), Franz Ulrich Theodor Aepinus (1724-1802) publizierten ihr mathematisches und auch physikalisches Werk großenteils lateinisch. Noch Albrecht von Haller (1707-1777) veröffentlichte seine ungezählten botanischen, anatomischen, physiologischen, medizinischen Arbeiten im Wesentlichen lateinisch, der nach ihm in Göttingen lehrende Johann Friedrich Blumenbach (1752-1840) schrieb lateinisch und deutsch nebeneinander. Immanuel Kant (1724-1804) dagegen verfasste seine Theorie des Himmels, der Mineraloge Abraham Gottlob Werner (1749-1817) seine Arbeiten über Gebirgsarten oder Fossilien, Chladni (1756-1827) seine physikalischen Arbeiten auf deutsch.

Die Schweden des 18. Jahrhunderts, Carl von Linné (1707-1778), Celsius (1701-1744), Torbern Bergman (1735-1784), Botaniker und Zoologen, Physiker, Mineralogen, publizierten in der Regel auf lateinisch, gelegentlich auch auf französisch oder in der Landessprache.

Im deutschen Sprachbereich gehen also die Wissenschaften vergleichsweise spät vom Lateinischen zum Deutschen über. Was bedeutet diese Verspätung? Wo lagen ihre Ursachen? Wie reagierte man darauf? Der Eindruck bleibt diffus. Es fehlte jede Voraussetzung für eine einheitliche Entwicklung, ein Staat, eine Hauptstadt wie in Frankreich oder England, oder wenigstens eine führende Region und ein einheitlicher Epochenwille wie in dem Norditalien der Renaissance. Der Dreißigjährige Krieg unterbrach und destruierte eine zaghaft beginnende neusprachliche Sachliteratur in Deutschland. Es fehlte eine Akademie.

Dieser Mangel und diese Lücke sind besonders von Leibniz empfunden worden. Das internationale Umfeld erzeugte einen Konkurrenzdruck; noch nicht einen politischen Nationalgedanken, wohl aber ein nationales Inferioritätsgefühl. Leibniz' Ermahnung an die Teutsche, ihren verstand und sprache beßer zu üben, sammt beygefügtem vorschlag einer Teutsch gesinten Gesellschaft von 1682/83 verdankt ihren Elan einem heftigen patriotischen, nationalen Impuls, Blackall (1966: 2) nennt sie zu Recht eine „patriotische Rhapsodie“. Leibnizens Absichten sind nationalpädagogisch, und er befindet sich dabei in Rivalität mit Frankreich und über Frankreich hinaus mit den Glanzzeiten der römischen und griechischen Kultur und Sprachkultur. In einer einheitlich ausgearbeiteten Sprache sieht er die Vorbedingung eines höheren Kulturniveaus in Deutschland. Eben deshalb ist ihm die deutsch-französische „Sprachmengerei“ seiner Zeit und die Sprachentrennung zwischen Gelehrten und Laien ein Stein des Anstoßes. Er knüpft an das patriotische Krisengefühl der Sprachgesellschaften an; hinter seiner Forderung einer deutschen Wissenschaftssprache steckt ein aufklärerischer Impuls, die Idee 
einer allgemeinen Aufklärung und Erziehung und des Austausches der geistigen Güter innerhalb der Nation. Sein Ideal ist das der Weltoffenheit und Weltläufigkeit. Es ist kein wissenschaftliches, sondern, wie im Fall des Thomasius, ein gesellschaftliches Ziel, das er verfolgt. Es zeigt sich an vielen Formulierungen, wie sehr ihm nach dem Vorbild der Französischen Akademie an der Hebung des allgemeinen Sprachgebrauchs, des „usage“, gelegen ist. Seine Empfehlung, eine deutsch-gesinnte Gesellschaft $\mathrm{zu}$ gründen und nachdenkliche, nützliche, auch annehmliche „Kernschriften“ in deutscher Sprache zu verfertigen - also einen Kanon der Sachprosa - steht im Dienst dieses Gedankens. Auch der Aufsatz Unvorgreifliche Gedancken, betreffend die Ausübung und Verbesserung der Teutschen Sprache (1697), der das Projekt einer Wörterbucharbeit in den Mittelpunkt stellt, denkt daran, dieses Projekt einer Gesellschaft oder Akademie anzuvertrauen. Der ursprüngliche Titel war Unvorgreifliche Gedancken betreffend die Auffrichtung eines Teutschgesinnten Ordens (vgl. zu diesem Abschnitt das Nachwort in Leibniz 1983).

Die Idee einer Akademiegründung hat Leibniz, der seit 1678 Mitglied der englischen und seit 1700 Mitglied der französischen Wissenschaftsakademie war, oft beschäftigt. Er betreibt eine Akademiegründung zum ersten Mal 1667 als 21jähriger, indem er vorschlägt, einer zu gründenden „Societas eruditorum Germaniae“ die Aufgabe zu übertragen, neben der Vermittlung eines gelehrten Briefwechsels und der Errichtung einer Bücherei sich insbesondere mit Medizin, Mathematik und Experimenten zu beschäftigen. - Es gab seit 1652 die „Academia Naturae Curiosum“, von Schweinfurter Ärzten und Naturforschern gegründet und später zu einer „Academia Caesarea Leopoldina“ erhoben (Leibniz 1916a: XXVIII; vgl. Heidelberger \& Thiessen 1981: 264), aber sie erlangte keine den ausländischen Akademien vergleichbare Bedeutung. Man könnte sagen: Leibniz führte die traditionelle Sprachkritik der Sprachgesellschaften des Barock, in deren Mittelpunkt die Pflege der Poesie stand, zusammen mit der Kritik an dem naturwissenschaftlichen Rückstand der Nation. Er hoffte für den deutschen Sprachbereich auf den Zusammenschluss der schon bestehenden Naturwissenschafts- und Sprachgesellschaften $\mathrm{zu}$ einer Akademie und sucht, durchaus im Sinne des staatlichen Nutzens und Prestigegewinns, Fürstenhäupter für seine Idee zu gewinnen. Bei der Gründung der Berliner „Sozietät [später „Akademie“] der Wissenschaften“ (1700), an der er maßgeblich beteiligt war, kam es zu einer solchen Vereinigung von Natur- und Geisteswissenschaften. Und wie Leibniz schon in seiner Denkschrift Eine deutschliebende Genossenschaft der Sprachkultur einen besonderen Raum zugemessen hatte, so heißt es hier in der von ihm mitverfassten General-Instruktion, die Akademie werde Sorge tragen, dass 
auch die uralte deutsche Hauptsprache in ihrer natürlichen, anständigen Reinigkeit und in ihrem Selbststand erhalten werde und nicht endlich ein ungereimtes Mischmasch und Undeutlichkeit daraus entstehe [...]. (Leibniz 1916a: 71; vgl. auch 58f.)

Wie wirkte es sich aus, dass eine deutsche wissenschaftliche Sachprosa sich erst verspätet entwickelte? Mit dem Übergang aus dem abgesonderten Latein in die öffentliche Bildungssprache geht notwendig ein breiter Entlehnungsschub aus den Wissenschaften in die Gemeinsprache einher. Die Grenzen zwischen Fachwelt und Laienpublikum, zwischen Fachsprache und allgemeiner Gebrauchssprache werden fließend, die wissenschaftlichen Inhalte können von nun an Teil der Allgemeinbildung werden, ihre Termini, in vielen Fällen der aus den Gelehrtensprachen beibehaltene griechische oder lateinische Fremdwortterminus, können Bestandteil der Gemeinsprache werden. Ist der verspätete Übergang ins Deutsche auch ein Grund dafür, dass die sog. „Fremdwörter“ und der Fremdwortterminus im deutschen Sprachbereich eine gewisse Sonderstellung behalten haben, dass sich eine gewisse Sprachenkluft zwischen Gelehrtenwelt und Laien erhalten hat?

Man darf freilich diesen Aspekt unsrer Sprachgeschichte nicht überbetonen. Wir müssen uns das lokal Gebundene, sozial Vielschichtige und geographisch Ungleichzeitige im deutschen Sprachgebiet des Spätmittelalters und der frühen Neuzeit vergegenwärtigen. Seit dem 13. Jahrhundert und vermehrt seit dem Buchdruck existiert, wie die eingangs erwähnte Pionierarbeit von Gerhard Eis und die seiner Schüler zeigt, eine umfangreiche und dicht verbreitete mathematische, naturkundliche, medizinische Sachprosa, ein sich im 16., 17., 18. Jahrhundert verbreiternder Strom von „Kunstbüchern“ und anderen Sachschriften, deren Wortschatz in den Lexika bisher nur lückenhaft gebucht ist und deren vollständige Erschließung das bisherige Bild von der Sprachgeschichte in dieser Zeit korrigieren wird. Wir müssen insbesondere nach dem Anlass fragen, nach dem sozialen Ort und der Schicht, in der sich der Übergang ins Deutsche vollzog.

\section{Die vierte Insel: Der praktische Anlass und soziale Ort des Übergangs ins Deutsche - zur frühen Geschichte einer deutschsprachigen Medizin und Mathematik und einer deutschsprachigen Universität seit Thomasius}

Vielleicht sollte man schon im Spätmittelalter von „Zwei Kulturen“ sprechen, von der theologisch gebundenen Kosmoslehre und der praktisch veranlassten Rat- 
geberliteratur. Edgar Zilsel (1976: 121f.) spricht in einer frühen Arbeit über Die sozialen Ursprünge der neuzeitlichen Wissenschaft von der sprachlichen und sozialen Barriere zwischen der „Handwerker-Literatur“ und der „Gelehrten-Literatur“. „Experimental-Wissenschaft konnte keine Existenz erlangen, bevor diese Barriere zerstört war.“

Die Anstöße zu einer Entwicklung und Verselbständigung der Naturwissenschaften kamen seit dem späten Mittelalter bekanntlich von außerhalb der Mauern der Universität und nicht zuletzt aus der Praxis. Auch der Anstoß zum Übergang in die Landessprache, in die Volkssprache kam aus der Praxis. Das beginnt, was neuerdings betont wird, bei den „artes mechanicae“ und den „artes magicae“ des Mittelalters und setzt sich fort in den Anstößen, die in der frühen Neuzeit von den Bedürfnissen der Lebenspraxis, Handwerk, Technik und bildender Kunst herkommen und dann auch vom Staat ausgehen, der aus Interesse an praktischer Nutzung z. B. die naturwissenschaftlichen Gesellschaften und Akademien fördert. Ein Blick auf die erhaltene Literatur aus den Bereichen der „artes mechanicae“ bestätigt den Eindruck eines praktischen Übersetzungsanlasses. Es gibt z.B. aus dem 1. Fach, dem Handwerk, Schriften für Färber, zur Alchemie, Bergbüchlein, aus dem 5. Fach, Wald und Tiere, Jagdliteratur, Büchlein zur Rossheilkunde, zum Angeln oder Fischen, aus dem 6. Fach, der Heilkunde, Arzneibücher, Kräuterbücher, Steinbücher, Syphilistraktate u.a.m. Die Schriften sind großenteils Übersetzungen aus dem Lateinischen, auch noch im 16. und 17. Jahrhundert. Ihre Absicht, irgendeine Art von „Kunst“ zu lehren, ihr praktischer Zweck, geht auch daraus hervor, dass ihre Grundform das Rezept ist, der Anweisungsstil der Ratgeber:

Swen die binen oder di wespen stechen, der sal uf die stat legen, da si in gestochen haben, alcativam unde girstin mel getempert mit ezzige.

Es gibt stereotype sprachliche Formeln, die jahrhundertelang überliefert werden (vgl. Eis 1967: 54, 58).

Wo kaufte man diese Art Literatur? Nicht nur im Buchhandel, der bis in die Mitte des 17. Jahrhunderts kränkelte. Daneben existierten „Bücherkrämer“, die auf Jahrmärkten verkauften, wandernde Kleinhändler. In den Leipziger Gerichtsakten ist das Warenlager eines solchen Händlers am 13. Oktober 1600 als herrenlos inventarisiert worden, darunter:

Funfzehn bund kleine Calender,

Eilff Traumbuchlein,

Acht Haus Apotecken,

Eine Historia vom Fausten,

Zwej Rechenbuchlein Johannis Albertj,

Sechs Kleine Kinder Catechismus. 
Das Lager des Besitzers David Böttner hatte einen Gesamtwert von 15 Talern. Man ersieht aus seinem Bestand sofort, wieso die deutschsprachige Anleitung, der Ratgeber, auf dem fliegenden Stand einen Ort hatte. Mancher von den wandernden Händlern oder Hausierern hatte ein „Lager“, das er, in Riemen geschnallt, auf dem Rücken tragen konnte (vgl. Kirchhoff 1889: 142-145).

Ein umstrittener Wanderarzt, der auch in Gasthäusern heilte und lehrte, zugleich ein überaus fruchtbarer Schriftsteller, war der schon erwähnte Theophrast Bombast von Hohenheim, genannt Paracelsus (1493-1541). Nach seiner mischsprachigen Basler Vorlesung von 1527 schrieb Paracelsus 1536 seine Grosse Wundartzney auf deutsch, und zwar deshalb, weil er für Bader und Wundärzte schrieb, in der Regel Unstudierte, die ihr Handwerk in einer Lehre lernten.

Ich habe [...] den Artzeten ein begnügen gethon, umb die anderen ist mir kayn sorg, ich hab [...] ein ländtlichen spruch gefürt, das mich kayner Rhetorick noch subtiliteten berümen kan, sonder nach der zungen meiner geburt, und landssprachen, der ich bin von Ainsidlen, des lands ein Schweytzer, soll mir mein lendtlich sprach niemandts verargen. ${ }^{17}$

Vermutlich hat Weimann die Originalität und Wirkung von Paracelsus überschätzt; Joachim Telle wie Gerhard Eis weisen darauf hin. - Aber es handelt sich doch immerhin um ein umfangreiches, verbreitetes, umstrittenes Schrifttum, das zeigt, wie Paracelsus lateinische Termini aufgreift oder neue bildet, das fremdsprachliche Vokabular assimiliert, übersetzt und neue deutsche Wörter erfindet: „Alkohol“ und „Gas“, „Homunculus“, „Kobalt“ und „Zink“, „Sylphe“ und „Undine“, „Aphorismen“, „alchimistisch“, „Attraktion“, „elektrisch“, „Elixier“, „Infektion“, „metallisch“, „Mikrokosmos“, „Natron“, „Paroxysmus“, „Signatur“, „Sublimierung“, „Tinktur“, aber auch Neubildungen aus dem Erbwortschatz wie „Amtsträger“, „Eiweiß“, „Erbkrankheit“, „Erkältung“, „Frauenkrankheit“, „Geisteskrankheit“ und „Seelenkrankheit“ haben sich nach Weimann durch ihn eingebürgert.

Unsere Kenntnis der Arzneibuchliteratur ist eine der Schneisen, die durch das Sachschrifttum des 16. bis 18. Jahrhunderts gelegt ist. Der Übergang ins Deutsche, der im späten Mittelalter beginnt und in der frühen Neuzeit auf breiter Basis fassbar ist, bleibt bis ins 18. Jahrhundert umstritten. Joachim Telle hat den Sprachenstreit im Überblick dargestellt und fasst die kontroversen Positionen zusammen:

Ihre besondere Würde verdanken diese Vor- und Frühformen medizinischer Aufklärungswerke dem ärztlichen Streben ihrer Urheber, durch Unwissenheit verursachtes Elend auf

17 Zitiert aus Der grossen Wundartzney, das Erst Buch [...] Doctors Paracelsi [...]. Augsburg 1536: Beschluszred. Zit. nach Hodermann 1891: 9. 
dem Gebiet laikaler Krankenversorgung zu lindern und mit Hilfe des Mediums Buch Menschen beizustehen, denen wirtschaftliche und/oder geographische Gründe den Gang zum Schulmediziner verwehrten. Insbesondere veranlaßten Mängel des Gesundheitswesens in ländlichen Gebieten, wo es an ausgebildeten Ärzten oder Apothekern am spürbarsten mangelte, den „gestreiffelten leyen“ von der Autorität des Arztes zu entbinden und zu befähigen, im Notfalle sein eigener Arzt, Barbierer und Apotheker sein zu können. Aufkommen und Fortentwicklung dieser vorab lesefähigen und mehrheitlich lateinkundigen Laien zugedachten, doch auch von medizinisch tätigen Menschen aller Ränge rezipierten Arzneibuchliteratur ließen viele Ärzte um das auf dem Sprach- und Wissensmonopol lateinkundiger Schulmediziner beruhende Medikalsystem fürchten und riefen anhaltende Widerstände hervor. [...] Bei der frühneuzeitlichen Kontroverse um den Gebrauch der Landessprache zur Formulierung medizinischen Lehrgutes hatten sich die Arzneibuchverfasser mit den Einsprüchen von Fachgenossen auseinanderzusetzen, die sich eine Verbesserung des Gesundheitswesens aus der Festigung einer nur von akademisch-lateinisch gebildeten Medizinern ausgeübten Arzneikunst erhofften und teilweise befürchteten, die landessprachige Verbreitung medizinisch-therapeutischen Wissens könne den sozialen Status ihres Standes bedrohen. Antworten auf den Hauptvorwurf der Lateiner, deutsche Arzneischriften reichten Kindern das Messer und begünstigten eine Medizin der „stümpler und hümpler“, finden sich allerorten. (Telle 1981: 90f.; auf breiter Materialbasis in Telle 1979)

Eine zweite Schneise, die durch den deutsch-lateinischen Wald der frühen Neuzeit gelegt ist, ist die Sprache der Mathematik. Deutschsprachige Rechenbücher und eine geometria deutsch gab es schon im Spätmittelalter; die praktischen Bedürfnisse sind deutlich erkennbar. Die sog. Geometria Culmensis, die erste geometrische Abhandlung in deutscher Sprache, war um 1400 zunächst im Auftrag des Hochmeisters Konrad von Jungingen in lateinischer Sprache verfasst und dann übersetzt worden. Die Schrift sollte der Feldmessung im Deutschordensland eine exakte Grundlage liefern. Sie enthält Ratschläge zur Vermessungstechnik und gibt Anweisungen zur Berechnung dreieckiger, viereckiger, kreisförmiger und kreisähnlicher Flächen. ${ }^{18}$

Die deutsche mathematische Fachsprache empfing frühe Impulse aus der Malerei und der Fassmesskunst. Albrecht Dürer veröffentliche 1525 eine Underweysung der messung mit dem zirckel un richtscheyt [...], eine Art Leitfaden für Malerknaben, der sie lehren sollte, Zeichnungen und Gemälde perspektivisch und geometrisch exakt zu behandeln. Die Schrift enthält klare knappe Konstruktionsbeschreibungen, die Beschreibung der Konstruktion eines regelmäßigen Fünfecks z.B., und eine neue deutschsprachige Terminologie.

An Stelle von „,conus“, „sphaera“, „cubus“ sagt Dürer „Kegel“, „Kugel“ und „Würfel“. Er verwendet kaum den an sich häufigen Typ der Lehnübersetzung, in dem das lateinische oder griechische Wort Teil für Teil nachgebildet wird. Für

18 Vgl. Schmitt 1972: 21f. sowie 19-21: 7. Arithmetik: Aus Friedrich Gerharts Practica. 
„diameter“ sagt er z.B. nicht „Durchmesser“, sondern „Zwerchlinie“, ,Querlinie“ also. Er bemüht sich um Neubildungen und zielt dabei auf Anschauung:

Die Elipsis will ich eine Eierlinie nennen, darum daß sie schier einem Ei gleich ist. Die Parabola sei genannt eine Brennlinie, darum so man aus ihr einen Spiegel macht, so zündet sie an. Aber die Hyperbola will ich eine Gabellinie nennen. ${ }^{19}$

Der Ansatz Dürers ist von Johannes Kepler systematisch ausgearbeitet worden, in einer deutschen Fassung seiner Nova Stereometria doliorum von 1615, einer Schrift, die dazu anleitete, den Rauminhalt von Fässern zu bestimmen. Kepler veröffentlichte diese seine Fassmesskunst (Messekunst Archimedis, 1616) auf deutsch, um die deutschen Behörden, Weinvisierer und Küfer anzusprechen. Er nennt auf dem Titelblatt des Buches, (das sich auch in der Freiburger Universitätsbibliothek befindet), seine Adressaten im einzelnen:

Allen und jeden Obrigkeiten/ Beampteten/ Kriegs Obristen/ Handelsleuten/ Buren= Müntz= $\mathrm{Bau}=$ und RechenMeistern/ Weinvisierern/ Hauswürthen/ und menniglichen in und ausser Lands/ fast dienlich: sonderlich aber den Kunst- und Antiquitetliebenden Lesern annemlich.

Aus diesem Weinvisierbuch, von dem er hofft, man werde es genießen beim österreichischen kühlen Wein, sind zahlreiche Termini in die mathematische Fachsprache eingegangen, terminologisierte Ausdrücke der Umgangssprache, aber auch Neubildungen: „Beweis“, „Gleichheit“, „ähnlich“, „kugelrund“, „Kegelschnitt“, „Querschnitt“, „Spirallinie“.

Es wäre auch aufschlussreich, der Frage nachzugehen, welche Ausdrücke nicht angenommen wurden und warum nicht: Kepler sagt für Ellipse ,ablenger Circkel“ oder „Ovallinie“, für Segment „Schnitz“, für Parallele „Gleichlaufende“, für Tangens „Anstreicher“, für cylinder „runde Seule“, für pyramis „zugespitzte Seule“.20

Die Schriften Dürers und Keplers hatten einen europäischen Erfolg in ihrer lateinischen Fassung; in der deutschen Version blieben sie vorläufig fast ohne

19 Zitiert nach Olschki 1919: 441. Ein Anhang dieses Bandes: 414-453, ist Dürer gewidmet. - Die drei theoretischen Schriften Dürers, alle im Uhl Verlag, Nördlingen, erhältlich, haben die Titel: Underweysung der messung mit dem Zirckel un richtscheyt, in Linien ebnen und gantzen corporen durch Albrecht Dürer zusammen getzogen [...], Nürnberg 1525; Etliche underricht, zu befestigung der Stett, Schlosz und Flecken, Nürnberg 1527; Hierin sind begriffen vier bucher von menschlicher Proportion, durch A.D. von Nürnberg erfunden und beschriben etc., Nürnberg 1528.

20 Vgl. hierzu den terminologischen Anhang von Keplers Werk und die Arbeiten von Busch 1933, Götze 1919 und Schirmer 1912. 
Wirkung. Der deutschsprachige Anschlag von Thomasius war zur Ostermesse von 1687 durchaus noch eine revolutionäre und eine folgenreiche Tat. Revolutionär war sie vor allem darum, weil sie eine deutsche Vorlesung im philosophischen Disziplinenbereich ankündigte. Thomasius war der erste, der Prinzipien, die aus dem ursprünglich verachteten Bereich der Realdisziplinen stammten, wo der Übergang ins Deutsche, wie das Beispiel Paracelsus zeigt, viel älter war, in den der philosophischen Disziplinen übertrug (vgl. Grimm 1983: 379). Es hat seinen Sinn, wenn sich der Übergang vom Gelehrtenlatein in die deutsche Wissenschaftssprache an seinen Namen heftet; denn es geschah vor einem grundsätzlichen und ausgearbeiteten theoretischen Hintergrund, und es hatte Erfolg. Thomasius hielt in Halle, nach seinem Weggang von Leipzig, weiterhin „lectiones publicas“ in deutscher Sprache, neben den üblichen lateinischen, obwohl es ihm durch preußische Kabinettsorder untersagt wurde; und schon 1711 ging man in Leipzig zu deutschen Kollegs über, weil man hier sonst seine Existenz gefährdet hätte (vgl. Thomasius 1970: 194).

Die wichtigsten europäischen Nachbarn waren, wie gesagt, auf diesem Weg vorangegangen, die Akademien Italiens, Frankreichs, Englands hatten den Übergang der Wissenschaft in die Landessprachen gefördert. Die Umschichtung in der Hierarchie der Kulturträger von der Universität zum Hof, vom humanistischen Gelehrtenideal zu dem der gesellschaftlichen und praktischen Weltklugheit, in der Frankreich um mehr als ein halbes Jahrhundert voraus war, legte auch in Deutschland eine Neudefinition der Aufgaben der Universität nahe. - Im Lande gab es überdies seit dem Spätmittelalter jenen Strom von „Kunstbüchern“ in den praktischen Fächern, gelehrte Ansätze zur deutschen Prosa, und es gab jene Entwicklung auf dem Buchmarkt, in den Lesegewohnheiten des Publikums, die einen solchen Übergang nahelegten. Die Schule war mindestens seit Mitte des 17. Jahrhunderts, unter dem Eindruck der Reformen Wolfgang Ratkes und Johann Balthasar Schupps, auf dem Weg zur Deutschsprachigkeit (vgl. z. B. Michel 1976: 185ff.; Matthias 1907: 47ff.).

Erst nach Thomasius erhielt eine deutsche Wissenschaftssprache eine breite Wirkungschance. Die mathematische Fachsprache ist dafür ein Beispiel; sie vermochte sich erst durchzusetzen als seriöse, anerkannte Sprache der Wissenschaft, als man an einigen protestantischen Universitäten zum Deutschen übergegangen war. Christian Wolff wurde ihr eigentlicher Begründer. Er führte eigene Termini ein, ordnete und definierte jedoch hauptsächlich das vorhandene Vokabular. Wolff war kein Purist. Er verwendete assimilierte Fremdwörter wie „Quotient“, „Quadrat“, „Proportion“ neben deutschen Wörtern wie „Zahl“, „Bruch“, „Nenner“, schuf einige Neubildungen, „Nebenwinkel“, „Brennpunkt“, „Spielraum“, „Versuch“, „Schwerpunkt“, „Hebel“, „Abstand“, „Geschwindigkeit“, und gebrauchte den einmal gewählten Terminus definitiv und konstant. 
Seine Anfangsgründe aller mathematischen Wissenschaften (1710) und sein $M a$ thematisches Lexikon (1734) waren grundlegend und wurden durch J.H. Lambert um die Mitte des 18. Jahrhunderts noch einmal durchgearbeitet und ausgebaut.

Kaum weniger wirksam war er durch seine neue wissenschaftliche Prosa, in der er sich von Poesie und Rhetorik so weit wie möglich trennt. Er definiert Begriffe, um mit ihnen als fest umrissenen Bausteinen $\mathrm{zu}$ arbeiten, schreibt emotionslos, systematisch und in der erklärten Absicht, die Begriffe kontextunabhängig, konstant und konnotatfrei zu verwenden, ihren latenten Bildgehalt auszublenden. Nichts darf der Phantasie überlassen bleiben, Wiederholung stört nicht, auch nicht die rhythmische Monotonie der einfachen Parataxe. Das Resultat ist, erstmals in deutscher Sprache, ein von überschaubaren Spielregeln geleiteter, klarer, methodischer Stil. Keine Redundanzen! „Meine Worte fallen, wie ich denke, und ich setze keines vergebens.“

Wolff ist zugleich ein Beispiel, dass die Durchsetzung einer Wissenschaftssprache abhängig ist von ihrer Eignung zur Schulbildung und von tatsächlicher Schulbildung. Seine entschiedene, definitive Sprache und Wirkung gehören offenbar zusammen; er übt Macht aus durch seine zahlreichen Schüler auf den Lehrstühlen des 18. Jahrhunderts (vgl. hierzu die entsprechenden Abschnitte bei Blackall 1966 und Busch 1933).

Der Streit zwischen den Verteidigern der lateinischen Gelehrtensprache und den Vorkämpfern der Volkssprache in den Wissenschaften ist aus verständlichen Gründen sehr heftig gewesen. Auch die Langsamkeit des Wandels ist verständlich; man spürte und erkannte die Tragweite dieses Vorgangs. Es ist erstaunlich, dass er so wenig untersucht worden ist.

Ich versuche Bilanz zu ziehen. Wie steht es mit Gewinn und Verlust?

Der Übergang in die Landessprache eröffnete neue Horizonte:

1. Das neue Recht, seine wissenschaftliche Weltsicht in der Muttersprache auszusprechen, ermöglichte es, aus den Mauern der lateinischen Überlieferung herauszutreten. Uralte Konventionen wurden hinfällig. Die Wissenschaft kam dem Leben näher.

2. Die Verpflichtung des wissenschaftlichen Autors, sein Argument in der Landessprache vorzutragen, demaskierte ihn, wenn er nichts zu sagen hatte und die Zunftsprache ihm lediglich als ,homerischer Nebel' (Leibniz) diente. Auch in dieser Hinsicht wurde die Wissenschaft demokratischer.

3. Die neue Bemühung, wissenschaftliche Beobachtungen und Überlegungen in der nationalen Mundart auszuarbeiten, brachte am Ende die bewunderte naturwissenschaftliche Prosa des 19. Jahrhunderts hervor. Wissenschaftliche Publikationen wurden eine Art Literatur.

4. Diese neue Literatur wurde zum Prüfstein und zur Herausforderung für die Belletristik. 
5. Der Abbau des Lateinischen legte - vorläufig - die Barriere zwischen Universität und Stadt, der gelehrten Welt und den Laien, nieder. Er machte das Wissen allgemein zugänglich.

6. Die den Wissenschaften entlehnten neuen Vokabeln und Begriffe erweiterten den Ausdruckshorizont der Umgangssprache - weit über die bisherige Reichweite hinaus.

7. Die Aufgabe der Zunftsprache übertrug das demokratisch gehandhabte Prinzip, überlieferte Wahrheit kritisch vor dem Richterstuhl von Vernunft und Erfahrung zu prüfen, in die Umgangssprache - machte das überlieferte Weltbild dadurch der öffentlichen Kritik zugänglich.

Aber die neuen Horizonte wurden mit beträchtlichen Unkosten geschaffen:

1. Die Einheit der europäischen Gelehrtensprache, der ,lingua Europaea universalis et durabilis ad posteritatem', die Leibniz erhalten wissen wollte, zerbrach.

2. Als Ergebnis wurde die Wissenschaft oft ein nationales, nicht selten nationalistisches Unternehmen. Schon der Übergang in die Landessprachen erscheint als eine Art Wettlauf in die Modernität.

3. Paradoxerweise lud die neue Öffnung der Wissenschaft sofort dazu ein, sie zu beaufsichtigen, zu manipulieren, auszubeuten und zu unterwerfen. Latein war ein Schutzwall gewesen, (wie der Fall Galileo Galilei zeigt).

4. Die Sprache der gelehrten Welt verlor ihren Charakter als handliches Werkzeug in einem von dem Leben abgesetzten Feld. Eine Kunstsprache, die zwar noch immer Sprache, aber von den Assoziationen und Konnotationen der Umgangssprache frei gewesen war, ging verloren.

5. Der Gelehrte, der zunehmend zum wissenschaftlichen Schriftsteller wird und im Zuge der Emanzipation von Theologie und Kirche, Poesie und Rhetorik die Aufgabe einer allgemeinen Weltinterpretation übernimmt, sieht sich von nun an einem Dilemma gegenüber. Jede moderne Sprache besteht aus zwei - (in unsrem Zusammenhang zwei!) - Codes, ,Dialekten“, die ein nahtloses Ganzes zu bilden scheinen, aber in Wirklichkeit verschiedene Fundamente haben. Das Ergebnis ist, dass der Gelehrte zwittrig wird, dass er nicht umhin kann, an ein allgemeines Publikum zu denken, wenn er für seine Fachgenossen schreibt. So werden seine Termini fast unvermeidlich zweideutig: sie werden als termini technici gewählt und hallen zugleich unvermeidlich in dem ganz anderen Register der Umgangssprache wieder. Sie verführen den Autor, ihre Wirkung zu berechnen, und lassen, ob er will oder nicht, schielende Begriffe entstehen. - Dafür, dass der Wissenschaftler zum Schriftsteller wird und die Grenze zwischen Fachwissenschaft und Populärwissenschaft fließend, ist Thomasius ein frühes Beispiel.

6. Wissenschaftliche Codes, die auf solche Weise konstruiert sind, haben schließlich die Neigung, das Alltagsleben zu überschatten und als Mythen zu 
herrschen. Sie werden zu brauchbaren Instrumenten und verändern, instrumentalisiert, ihren Charakter: aus Hypothesen werden Doktrinen. Die Lehren und ,Sprachen'von Marx, Darwin, Freud sind krasse Beispiele dafür.

7. Die Umgangssprache wird zunehmend durch ein nur halb verstandenes wissenschaftliches Vokabular belastet. Dies Vokabular befestigt, mehr als die überlieferte Gemeinsprache es vermag, Mythen. Es überschattet das einfachste Gespräch und nimmt ihm seinen konkreten, persönlichen Sinn.

Richard Hodermann berichtet in seiner Dissertation Universitätsvorlesungen in deutscher Sprache um die Wende des 17. Jahrhunderts (1891) von einer Rede, die 1837 in Jena gehalten wurde. Der Redner war Heinrich Karl Abraham Eichstädt, sein Thema war Thomasius, und er hielt diese Rede 150 Jahre nach dem Anschlag des deutschen Programms auf lateinisch.

Latein hält dieser Philologe Eichstädt für die einzig mögliche Sprache des Gelehrten, und Hodermann (1891: 39) bemerkt dazu etwas, was aufhorchen lässt, weil man eine menschliche Stimme hört. Er schreibt:

[...] es beschleicht uns eine sonderliche Freude, in einer Rede aus dem Jahre 1837 noch solcher veralteten, verspäteten Ansicht über die That unseres Thomasius zu begegnen - so findet man wohl auch einmal auf frisch gepflügtem Ackerboden eine alterthümliche, verwischte, blinde Thonscherbe, nach der man sonst tief in unterster Schicht zu graben hat, man hebt sie auf und freut sich lächelnd dankbar des unverhofften eigenartigen Fundes. 